\title{
INFLUENCE DE L'INGESTION DE PROTÉINES IODÉES SUR LES RÉSERVES VITELLINES DE L'OVOCYTE CHEZ LA POULE DOMESTIQUE
}

PAR

\author{
L. LACASSAGNE $\left({ }^{1}\right)$ \\ Station de Recherches avicoles \\ Centre national de Recherches zootechniques \\ Jouy-en-Josas
}

I.es travaux destinés à étudier l'action de la thyroxine (distribuée pure ou sous forme de protéine iodée ou de poudre de thyroïde desséchée) sur la ponte sont très nombreux. Malgré de multiples différences de conditions expérimentales, il semble que les résultats puissent se résumer comme suit :

Chez les poules de deux ans et plus, le léger hyperthyroïdisme provoqué par l'ingestion de protéines iodées augmente la ponte soit par une meilleure intensité (TURnER et col. I945 $a$, I946, Oloufa, I954), soit par un allongement de la période de ponte (Turner et col. I945 $b$, I948, I949).

Chez les poulettes ayant I4 mois ou moins en début d'expérience un traitement similaire n'a aucune influence sur le nombre d'œufs pondus (Asmundonon et Pinsky, I935 ; Charlet't-Lerry, Leroy, Firançors, I953; Delipon, I956 ; Godfrey, I949 ; LiLlite, Sizemore, Mirligan et Bird, I952; Savage, Turner, Kempster et Hogan, 1952 ; Hutt et GOWE, I948). Ces deux derniers auteurs remarquent un fléchissement de la ponte durant les huit premières semaines de traitement. De même, BERG et BEARSE I95I, obtiennent une chute de production durant les ro premières semaines de leur expérience. Cependant, Turner et col. I945 $b$, remarquent dans les mêmes conditions une augmentation de production par retard du déclin saisonnier de la ponte.

Seuls de tous ces auteurs Asmundson 1931, Asmundson et Pinsky I935, mentionnent une diminution du poids de vitellus contenu dans chaque ovule après ingestion de poudre de thyroïde desséchée, sans changement de la production totale. Pour la simplification du texte, nous appelons ovule tout ovocyte détaché de l'ovaire et ceci depuis la

(1) Avec la collaboration technique de Mue Gate et M. GrLiet. 
rupture du follicule jusqu'à la ponte de l'œuf, que la fécondation soit intervenue ou non durant cet intervalle.

A la suite de ces travaux il nous a paru intéressant :

- de chercher s'il se produit un abaissement des réserves vitellines de chaque ovule chez des animaux soumis à des doses de thyroxine égales à celles recommandées par TURNER et col. I945 a et généralement employées dans les travaux se rapportant à l'étude de l'action des protéines iodées sur la ponte.

- dans 1'affirmative de vérifier ultérieurement si cet abaissement est compatible avec le maintien de la production ou est à 1'origine d'un fléchissement de la ponte tel que l'ont observé HuTT et GowE I948 et BERG et BEARSE I95I. Cela afin de mettre en évidence les différentes régulations susceptibles de se produire au niveau du follicule en vue du maintien de l'ovulation et d'apporter quelques éléments nouveaux à l'étude de l'interaction ovogénèse-ovulation.

\section{METHODE}

Huit poules Rhode $\times$ Wyandotte nées à la même date et âgées de Io mois au début de l'expérience furent séparées en 2 lots, un témoin et un traité recevant une ration contenant 0,044 p. Ioo de caséine iodée à $I, 3$ p. roo de dl thyroxine. Les aninuaux traités ingéraient ainsi en moyenne chaque jour $220 \mathrm{ug}$ de thyroxine par kilogramme de poids vif.

Pendant la durée de l'expérience les animaux furent conditionnés en lumière de façon à leur fournir I2 heures d'éclairement. Pour chacun des lots la période de base fut de trois semaines ainsi que la période de traitement, ces deux périodes de $2 \mathrm{I}$ jours étant séparées par un intervalle de 48 heures pris comme période de transition.

L,e poids de chaque ovule fut évalué par pesée directe après ablation des chalazes et essuyage complet pratiqué en faisant rouler le jaune sur une feuille de papier légèrement absorbant de manière à amener tous les points de sa surface au contact du papier. Cette méthode, déjà employée par BASTIAN et ZARROW I955, constitue un moyen précis d'évaluer le poids de vitellus à l'état frais. L'erreur due à l'évaporation est inférieure à $0,2 \mathrm{p}$. I ooo pour une durée de pesée de I minute. I,es erreurs dues à l'essuyage sont également négligeables : un double essuyage, une chalaze non enlevée, manipulations jamais effectuées, entraînent une erreur maximum de 4 p. I ooo.

Les ovules accidentés ne figurent pas aux tableaux I, II, III mais sont représentés au tableau IV par la moyenne des ovules de la poule correspondante durant la période considérée. De plus, les chiffres qui figurent aux tableaux I, II, III, ainsi que l'estimation de la sécurité des moyennes des différents lots proviennent de calculs effectués sur 
un nombre d'œufs identique pour chaque poule de façon à éviter les erreurs qu'introduirait pour un lot une ponte différente par poule, étant donné que dès le début, le poids des ovules diffère avec chaque animal.

\section{RÉSULTATS}

Durant la période de base aussi bien que durant la période de traitement, la ponte est demeurée sensiblement la même.

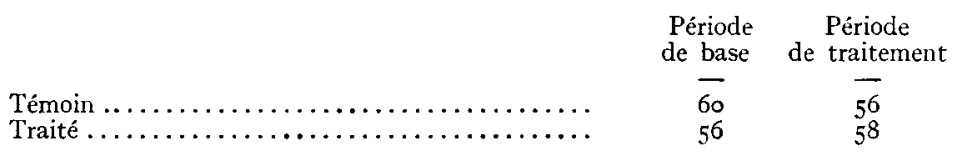

\section{Evolution des réserves vitellines}

\section{Io Analyse par lot}

Les résultats résumés dans le tableau ci-dessous montrent que la caséine iodée, à la dose indiquée, abaisse le poids moyen de chaque ovule.

\section{TABLEAU I}

\begin{tabular}{|c|c|c|c|c|c|}
\hline & \multicolumn{2}{|c|}{ Période de base } & \multicolumn{3}{|c|}{ Période de traitement } \\
\hline & $\begin{array}{l}\text { Nombre } \\
\text { d'eufs }\end{array}$ & $\begin{array}{l}\text { Poids moyen des } \\
\text { ovules en } g\end{array}$ & $\begin{array}{l}\text { Nombre } \\
\text { d'œufs }\end{array}$ & $\begin{array}{l}\text { Poids moyen des } \\
\text { ovules en } g\end{array}$ & Différence \\
\hline $\begin{array}{l}\text { Lot témoin . } \\
\text { Lot traité .. }\end{array}$ & $\begin{array}{l}52 \\
44\end{array}$ & $\begin{array}{l}I 8,54 \pm 0,18 \\
19,83 \pm 0,20\end{array}$ & $\begin{array}{l}40 \\
48\end{array}$ & $\begin{array}{l}\mathrm{r} 9,43 \pm 0,20 \\
\mathrm{r} 9,65 \pm 0, \mathrm{r} 5\end{array}$ & $\begin{array}{l}+\quad 0,89 \\
-0,18\end{array}$ \\
\hline
\end{tabular}

La différence entre moyennes pour le lot témoin avant et pendant la période de traitement est égale à $+0,89 \mathrm{~g}$ et est significative au seuil de I p. Ioo. Inversement, la différence entre moyennes pour le lot traité avant et pendant la distribution de thyroxine est égale à - $0, \mathrm{I} 8$ et n'est pas significative. En fait, dans la deuxième phase expérimentale se rapportant au lot traité, nous devons considérer deux périodes : une première période durant laquelle les ovules pondus présentent des couches de vitellus qui n'ont pu être atteintes par le traitement. Sa durée est environ de Io jours, dont 9 jours de temps moyen de développement d'un ovocyte dans sa phase de grand accroissement et un jour correspondant à la formation de l'œuf dans l'oviducte.

Enfin, une detuxième période durant laquelle les ovules pondus ont pu subir le traitement dans la totalité de leurs réserves vitellines.

Nous prendrons comme limites de la première période les dates du 27 mars au 6 avril inclus. L'examen des graphiques I et 2 nous dé- 

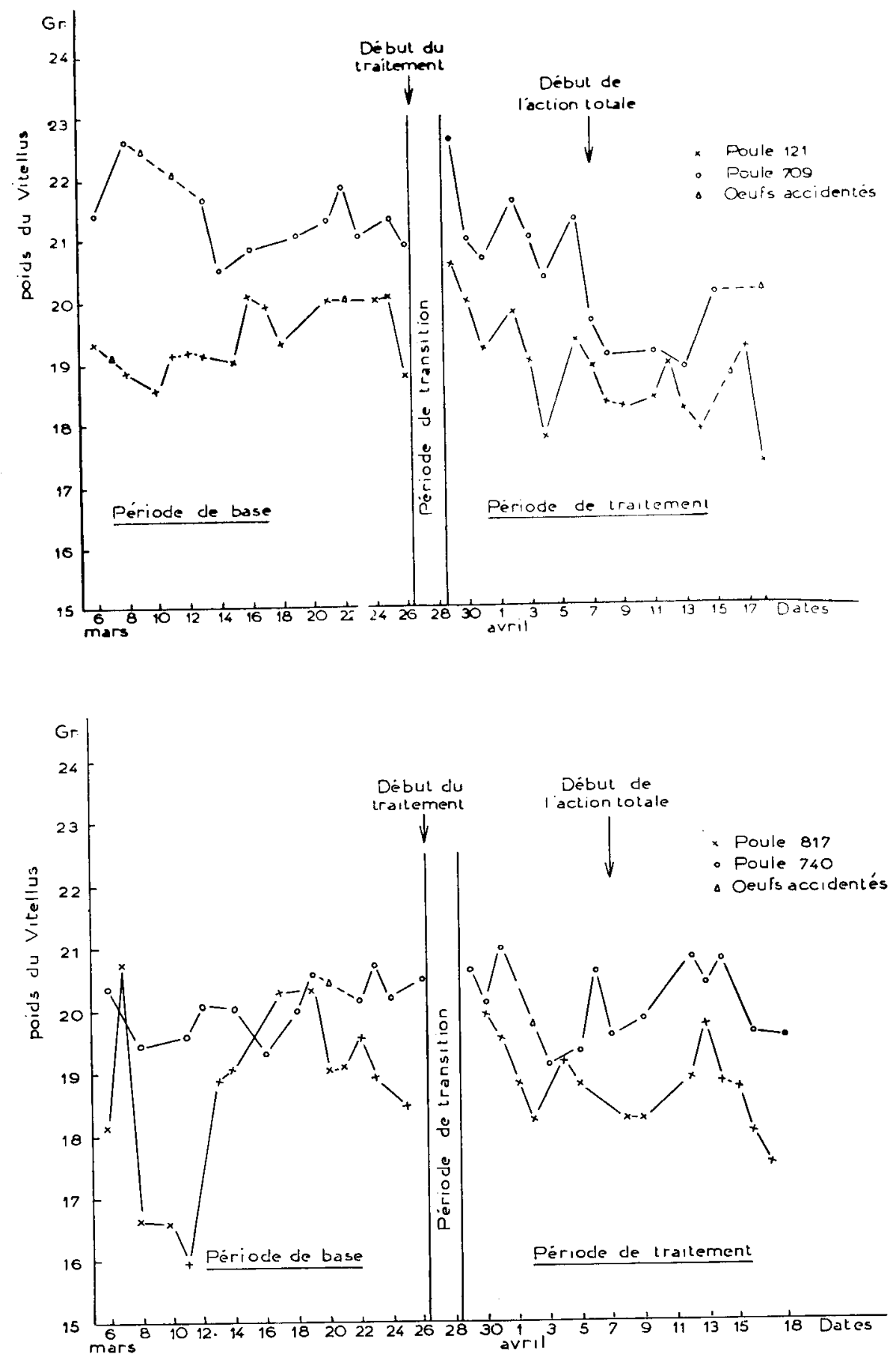

Graphioques I et II. - Courbes d'évolution des réserves vitellines de chaque ovule en fonction du temps chez les animaux traités à la caséine iodée. 
montre que la période ainsi définie correspond à la durée réelle d'évolution des ovocytes dans le cas de l'expérience. La deuxième période s'étend donc du 7 au I8 avril inclus et donne les résultats suivants.

TABLEAU II

\begin{tabular}{|c|c|c|c|c|c|}
\hline & \multicolumn{2}{|c|}{ Période de base } & \multicolumn{3}{|c|}{$2^{e}$ période de traitement } \\
\hline & $\begin{array}{l}\text { Nombre } \\
\text { d'oeufs }\end{array}$ & $\begin{array}{l}\text { Poids moyen des } \\
\text { ovules en } g\end{array}$ & $\begin{array}{l}\text { Nombre } \\
\text { d'œufs }\end{array}$ & $\begin{array}{l}\text { Poids moyen des } \\
\text { ovules en } \mathrm{g}\end{array}$ & Différence \\
\hline Lot traité $\ldots . . .$. & 44 & $\mathrm{I} 9,83 \pm 0,20$ & 20 & $19,25 \pm 0,19$ & $-0,58$ \\
\hline
\end{tabular}

Cette différence de $-0,58$ est significative au seuil de $5 \mathrm{p}$. Ioo. La moyenne de poids de vitellus s'est donc abaissée durant le traitement. Or, nous savons que le poids de l'ovule croît continuellement à partir de la date d'entrée en ponte et beaucoup plus vite en début de ponte qu'en fin de ponte. Dans le cas qui nous préoccupe chaque période expérimentale étant égale à 2I jours, nous pouvons supposer que sur un laps de temps aussi court l'augmentation de poids de l'ovule est une fonction linéaire du temps. Nous obtenons ainsi quatre droites de régression (voir graphique $n^{0} 3$ ) dont les équations figurent au tableau ci-après :

\section{TABLEAU III}

\begin{tabular}{|c|c|c|}
\hline & Période de base & Période de traitement \\
\hline $\begin{array}{l}\text { Lot témoin } \ldots \ldots \ldots \\
\text { Lot traité } \ldots \ldots \ldots\end{array}$ & $\begin{array}{l}\hat{y}=0,046 x+18,22(1) \\
\hat{y}=0,069 x+19,41(2)\end{array}$ & $\begin{array}{l}\hat{y}=+0,076 x+19,01 \\
y=-0,114 x+20,39\end{array}$ \\
\hline
\end{tabular}

$y$ représente le poids de l'ovule et $x$ le numéro d'ordre de l'œuf. L,e calcul montre que les régressions (I), (2), (3) sont effectivement linéaires.

La représentation graphique de ces équations fait apparaître la brusque décroissance du poids de vitellus pendant la distribution de thyroxine. Décroissance mise en valeur par le fait que les premiers oufs pondus ne sont peu ou pas affectés par le traitement contrairement aux œufs pondus dans la deuxième période.

Après examen du graphique no 3 , il devient évident que dans le lot traité la différence des moyennes de poids de vitellus des ovocytes détachés de l'ovaire avant et pendant la distribution de thyroxine est beaucoup plus grande qu'il n'apparaît aux tableaux I et II. Il faut en effet comparer la moyenne obtenue pendant le traitement à la moyenne qui aurait été atteinte sans lui ; le calcul ainsi fait nous donne une différence virtuelle supérieure à I $\mathrm{g}$. 


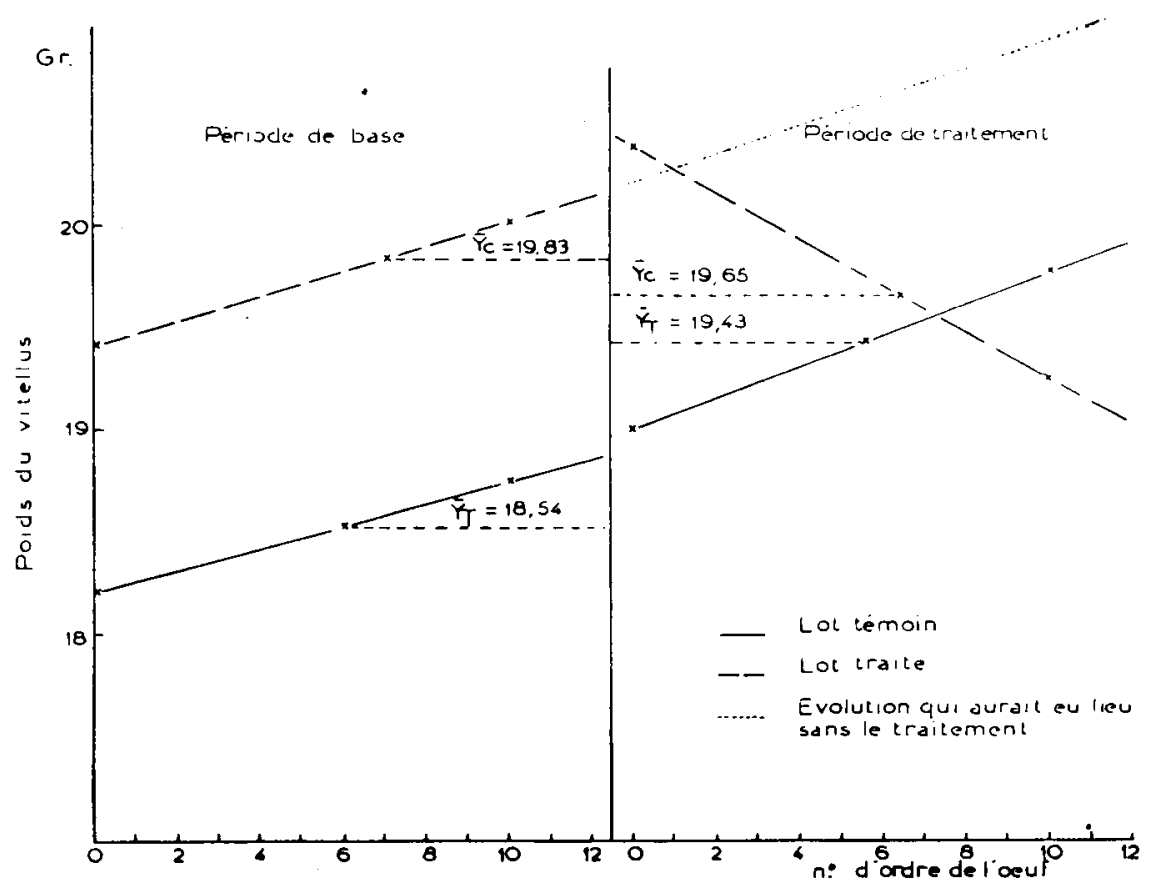

GRAPHIQUE III. - Droites de régression représentant l'évolution des réserves vitellines de chaque ovule en fonction du numéro d'ordre de l'ouf qui le renferme.

\section{$2^{\circ}$ Analyse par animal}

Au tableau $\mathrm{n}^{0} 4$ nous avons comparé la moyenne du poids des ovules pondus par une poule durant la deuxième période de traitement ainsi que la quantité totale de vitellus exportée par l'animal durant ce même temps, aux valeurs correspondantes obtenues pendant les douze

'TABLEAU IV

\begin{tabular}{|c|c|c|c|c|c|c|c|c|}
\hline \multirow{2}{*}{$\begin{array}{l}\text { Numéro } \\
\text { des poules } \\
\text { traitées }\end{array}$} & \multicolumn{3}{|c|}{$\begin{array}{l}\text { Période de base } \\
\text { I } 2 \text { derniers jours }\end{array}$} & \multicolumn{3}{|c|}{$2^{\mathrm{e}}$ période de traitement } & \multirow{2}{*}{$\begin{array}{l}\text { Diffé- } \\
\text { rence } \\
\text { poids } \\
\text { moyen } \\
\text { ovules } \\
\text { en g }\end{array}$} & \multirow{2}{*}{$\begin{array}{l}\text { Diffé- } \\
\text { rences } \\
\text { significa- } \\
\text { tives }\end{array}$} \\
\hline & $\begin{array}{l}\text { Nbre. } \\
\text { d'œufs }\end{array}$ & $\begin{array}{c}\text { Vitellus } \\
\text { total } \\
\text { exporté } \\
\text { en } g\end{array}$ & $\begin{array}{c}\text { Poids } \\
\text { moyen } \\
\text { des ovules } \\
\text { en g }\end{array}$ & $\begin{array}{c}\text { Nbre } \\
\text { d'œufs }\end{array}$ & $\begin{array}{l}\text { Vitellus } \\
\text { total } \\
\text { exporté } \\
\text { en } g\end{array}$ & $\begin{array}{c}\text { Poids } \\
\text { moyen } \\
\text { des ovules } \\
\text { en } g\end{array}$ & & \\
\hline $\mathrm{r} 2 \mathrm{I}$ & 9 & I 76,78 & $\begin{array}{r}19,64 \\
\pm \quad 0,17\end{array}$ & Io & I $8,3,60$ & $\begin{array}{r}18,36 \\
\pm 0,17\end{array}$ & $-1,28$ & I P. \\
\hline 709 & 7 & $r_{4} 8,29$ & $\begin{array}{r}21,18 \\
\pm \quad 0,13\end{array}$ & 6 & І I $6, \mathrm{I} 2$ & $\begin{array}{r}19,35 \\
\pm \quad 0,17\end{array}$ & $-\mathrm{I}, 83$ & $\begin{array}{c}\grave{a} \\
\text { I p. } 1000\end{array}$ \\
\hline $74^{\circ}$ & 8 & 161,63 & $\begin{array}{r}20,20 \\
\pm 0,15\end{array}$ & 7 & $\mathrm{I} 40,74$ & $\begin{array}{r}20,1 \mathrm{II} \\
\pm 0,23\end{array}$ & $-0,09$ & $\begin{array}{c}\text { non } \\
\text { signif. }\end{array}$ \\
\hline $8 \mathrm{r} 7$ & 7 & $\mathrm{I} 35,79$ & $\begin{array}{r}19,40 \\
\pm \quad 0,28\end{array}$ & 8 & $\mathrm{I} 48,3 \mathrm{I}$ & $\begin{array}{r}18,54 \\
\pm \quad 0,25\end{array}$ & $-0,86$ & $5 \mathrm{p}$ ioo. \\
\hline Total & $3 I$ & 622,49 & & $3 \mathrm{I}$ & 588,77 & & & \\
\hline
\end{tabular}


derniers jours de la période de base. L,es chiffres représentant la quantité de vitellus exportée en I2 jours ne sont qu'une approximation de la quantité de vitellus accumulée dans l'ovaire durant ces mêmes phases de la ponte. En effet, ces valeurs ne représentent réellement les quantités sécrétées que si la production quotidienne de vitellus demeure constante avant et après la période de contrôle durant un nombre de jours égal à celui des périodes étudiées, ce qui n’est pas.

La réduction moyenne du poids de vitellus par ovule par rapport à la période de base est donc de :

6,5 p. IOo pour la poule I2I

8,6 p. Ioo pour la poule 709

0,4 p. roo pour la poule 740

4,4 p. Ioo pour la poule $8 \mathrm{I} 7$.

Chaque animal répond donc de façon différente à un même taux de thyroxine incorporée dans la pâtée. Ce résultat peut être rattaché soit à une ingestion plus ou moins importante de nourriture, soit à une plus ou moins bonne utilisation digestive de la thyroxine contenue dans l'aliment, soit à un taux de thyroxine endogène différent, soit à une sensibilité inégale à un même taux de thyroxine. Pour l'ensemble du lot, la différence de quantité de vitellus exportée durant les I2 derniers jours de la période de base et les I 2 derniers jours de la période de traitement est de $-33,72 \mathrm{~g}$ soit une diminution de 5,4 p. Ioo pour un nombre d'orulations apparent demeuré identique. En fait, il est impossible d'affirmer que ce nombre n'a pas subi de changement étant donné que sur une aussi courte période une différence de un jour dans la durée d'expérience peut introduire une erreur voisine de $5 \mathrm{p}$. Ioo par simple fait que la ponte est constituée de séries.

Les travaux antérieurs ne peuvent également lever cette incertitude et seule la détermination de la durée d'évolution du follicule peut nous apporter une réponse définitive. Asmundson et Pinsky 1935, ont montré que la réduction du poids de l'ovule correspond à un taux moindre de dépôt quotidien de vitellus dans le follicule ce qui les amène à conclure que la durée d'évolution du follicule doit demeurer inchangée. Cette conclusion demande à être vérifiée par une méthode directe car admettre que le follicule met le même temps pour arriver à maturité, le taux d'ovulations demeurant inchangé, c'est admettre que la réduction de la vitellogenèse totale est proportionnelle à la réduction par ovule.

\section{RESUME}

La distribution à des poules âgées de ro mois, de protéines iodées, renfermant $\mathrm{I}, 3 \mathrm{p}$. Ioo de d1 thyroxine, à la dose de $0,044 \mathrm{p}$. Ioo dans la ration, fait apparaittre au cours des trois premières semaines de traitement: 
- une inhibition de l'augmentation du poids des ovules qui se serait normalement manifestée durant cette période ;

- un abaissement significatif du poids moyen des ovules si 1'on se réfère aux derniers jours de la période de base.

La réduction moyenne de poids ainsi obtenue est pour l'ensemble du lot de $5,4 \mathrm{p}$. Ioo et prend une valeur supérieure à ce chiffre si l'on se réfère non plus à la période de base mais à l'évolution normale qui aurait eu lieu sans le traitement.

Reçu pour publication le 6 avril $\mathrm{x} 957$.

\section{RÉFÉRENCES BIBLIOGRAPHIQUES}

Asmundson (V. S.). - Effect of hormones on the formation of the hen's egg. Poul. Sci., 10, I57-I65, I93I.

Asmundson (V. S.) and Pinsky (P.). - The effect of the thyroid on the formation of the hen's egg. Poul. Sci., 14, 99-I04, I935.

Bastian (J. W.) and Zarrow (M.X.). - A new hypothesis for the asynchronous ovulatory cycle of the domestic hen (gallus domesticus). Poul. Sci., 34, 776-788, I955.

BERG (L. R.) and BEARSE (G. E.). - Effect of iodinated casein and thiouracil on the performance of laying birds. Poul. Sci., 30, 2I-28, I95I.

Charlett-Léry (G.), François (A. C.) et Leroy (A. M.). - Effet de l'ingestion de protéines iodées sur la ponte et sur la teneur en vitamine A des ceufs de poule. Annales I.N.R.A., I59-I6I, I953.

DELPON (Yves). - Action des protéines iodées sur la croissance et sur la ponte. Thèse vétérinaire, 1956 .

GODFREY (G. F.). - The effect of feeding thyroprotein on egg shell quality and hatchability. Poul. Sci., 28, 867-873, I949.

HutT (F. B.) and Gowe (R. S.). - On the supposed effect of iodocasein upon egg production. Poul. Sci., 2\%, 286-293, I948.

LiLlite (R. J.), Sizemore (J. R.), Mri,igaAn (J. I.) and Bird (H. R.). Thyroprotein and fat in laying diets. Poul. Sci., 31, I037-I042, I952.

Oloufa (M. M.). - Influence of thyroprotein and darkness on egyptian chicken during summer. Poul. Sci., 33, 649-652, I954.

Savage (J. E.), Turner (C. W.), Kempster (H. L.) and Hogan (A. G.). The effects of vitamin BI2 and thyroprotein on egg production, egg weight, shell quality and hatchability. Poul. Sci., 31, 22-3I, I952.

TURNer (C. W.), IRWIN (M. R.) and ReINEkE (E. P.). - Effect of the thyroid hormone on egg production of white Leghorn hens. Poul. Sci., 24, I7I-r80, I945 a.

Turner (C. W.), Kempster (H. L.), Hali (N. M.) and Reineke (E. P.). - The effect of thyroprotein on egg production. Poul. Sci., 24, 522533 , I945 b.

Turner (C. W.), Kempster (H. L.) and Hali (N. M.). - Effect of continued thyroprotein feeding on egg production. Poul. Sci., 25, 562-569, I946.

Turner (C. W.) and Kempster (H. L.). - Thyroprotein feeding to 7 year old hens. Poul. Sci., 28, 826-829, I949. 